\title{
Conversation analysis of doctor-patient encounters in general practice clinics - an overview
}

\author{
SØREN BECK NIELSEN
}

In the last couple of decades conversation analysis (CA) has evolved to become a substantial academic method for conducting workplace studies. Interaction in a rich variety of institutional settings has been investigated, but most attention has probably been paid to clinical settings, notably doctor-patient encounters in general practice clinics. These studies are discussed in this paper and an overview of the many findings is provided. It is argued that five distinct, but often interrelated, analytical approaches are found: sequential analyses of local organization, sequential analyses of global organization, multimodal studies, comparative studies and quantitative studies. Whereas the first approach forms the core of conversation analytic studies, the remaining four are later developments. In total the findings contribute an in depth understanding of the interactional aspects of doctor-patient encounters: they show the extent to which doctors and patients attentively and jointly co-coordinate their actions at every moment during the encounters. 